\title{
Natural Cycle IVF can be a successful treatment alternative for patients with low ovarian reserve
}

\author{
Markus Nitzschke, MD, Sonny J. Stetson, PhD, Luis A. Ruvalcaba, MD \\ Instituto Mexicano de Infertilidad (IMI), Centro Medico Puerta de Hierro \\ Zapopan (Guadalajara), Jalisco, Mexico \\ Accredited Redlara center
}

\section{ABSTRACT}

Objective: In general, patients with low ovarian reserve are difficult to manage and have a relatively poor prognosis. Using traditional stimulation protocols like the long $\mathrm{GnRH}$ agonist protocol or the $\mathrm{GnRH}$ antagonist protocol in these patients often results in low ovarian response and poor pregnancy rates. In natural menstrual cycles without ovarian stimulation, every month the female body naturally selects the best possible oocyte for ovulation. Nevertheless, hormonal changes due to ovarian insufficiency can influence and change the individual menstrual cycle pattern of each patient over the time, which may result in difficulties to conceive naturally. We observed the menstrual cycle pattern of patients with low ovarian reserve in order to distinguish different stages of ovarian insufficiency. Once we were able to describe the different stages, we developed new treatment approaches for each patient group based on Natural Cycle IVF.

Methods: In 2012, the menstrual cycle pattern of 10 patients with $\mathrm{AMH}<1.0 \mathrm{nmol} / \mathrm{L}$ were observed. Patients were 22 42 years old (average 39.3). Blood samples were drawn to determine $\mathrm{AMH}, \mathrm{FSH}, \mathrm{LH}, \mathrm{E} 2$ and transvaginal ultrasound scans were performed on different days of the cycle. Depending on the cycle pattern of each patient, we offered individualized treatment approaches based on Natural Cycle IVF using Clomifen citrate to control ovulation, GnRH agonists to induce ovulation and either Ethinyl-Estradiol or combined oral contraceptive pills to regulate the cycle. Embryos were vitrified in day 2 stages and transferred later in artificial cycles. Patients were informed about off label use of the medication and informed consents were signed.

Results: Based on our observation, we could describe four different stages of ovarian insufficiency. We were able to perform oocyte retrievals and embryo transfers in all 10 patients. A total of 33 natural cycles were initiated. Premature ovulation occurred in 3 cycles $(9.0 \%)$ and no retrieval was attempted. Among the attempted 30 oocyte retrievals, $21(70.0 \%)$ were successful. Out of those 21 oocytes 11 (36.6\% per retrieval) were mature and 10 (33.3\% per retrieval) were immature. ICSI resulted in 8 fertilizations $(72.7 \%$ per mature oocyte). Out of 8 transfers, $3(37.5 \%)$ resulted in biochemical pregnancy. Two patients delivered $(25.0 \%)$, one patient had a miscarriage at 8 weeks of pregnancy.

Conclusion: Our experience shows that ovulation can successfully be controlled by the use of Clomiphene citrate and does not necessarily require $\mathrm{GnRH}$ analogues for pituitary suppression. This knowledge opens new space for development of alternative protocols respecting the patients' own physiology with no need for heavy stimulation. Patients with ovarian insufficiency may benefit from this approach, which can be offered before referring them to egg donation.

\section{INTRODUCTION}

For the last 30 years, the so-called "long gonadotropin releasing hormone agonist $(\mathrm{GnRHa})$ pituitary suppression regimen" with relatively high doses of exogenous follicle combined-stimulating hormone (FSH) remains the most frequently used stimulation protocol. The success of this protocol is mostly based on the fact that pituitary suppression and exogenous ovarian stimulation can be easily learned and reproduced. These artificial cycles are predictable and easy to schedule, which makes IVF practicable for clinics all over the world. A deeper understanding of each patient's particular cycle pattern is not really necessary. Over the time the knowledge to observe the patient's cycles and to learn from them, has been lost. Although the "long GnRHa pituitary suppression regimen" works very well in many patients, there are certain patient groups, which are difficult to treat. For example, patients with low ovarian reserve and high basal FSH may not respond sufficiently to ovarian stimulation and their treatment cycles are often canceled. So far, the lack of deeper understanding of ovarian physiology has prevented the development of alternative treatment approaches for these patient groups. Yet another stimulation protocol for IVF, which has recently been described by Teramoto (Teramoto \& Kato, 2007), involves the use of $50 \mathrm{mg}$ Clomiphene citrate from cycle day 3 onwards. This new protocol is designated as "minimal ovarian stimulation". It is not designed for women with low ovarian reserve in particular. Clomiphene is administered in this method for a relatively long period of time, i.e. 10-12 days until the day before maturation is triggered by administration of a GnRH agonist. Oocytes are then retrieved 32-35 h later. By this method Teramoto makes use of the antagonistic action of Clomiphene citrate to the estradiol receptor on the hypothalamus level, inhibiting both positive and negative feedback, and resulting in the induction of the ovarian stimulation and suppression of ovulation.

In order to develop treatment solutions for "difficult" patients, our group used the most empirical approach possible. We went back to pure observation of each patient's cycle, trying to understand her individual hormonal pattern. Based on this observation, we classified different types of ovulation. In a second step, we used an adapted treatment approach on each ovulation group. The different treatments were based on our personal experience and different approaches described in the literature, mainly those of Teramoto (Teramoto \& Kato, 2007). Natural Cycle IVF could successfully treat most patients. The only medication we used in these cases was Clomifen citrate (CC) to control ovulation. In other patients with ovulation disorders, we successfully applied Ethinyl-Estradiol (EE), combined oral contraceptive pills (COCP) or Clomiphene citrate depending on the pathology. The following observations and description of protocols are not evidence based and rely only on the empiric experience of our group. Nevertheless, using our approach, we managed to have pregnancies and life births in nearly menopausal women and other "hopeless" cases, which were originally referred to egg donation.

\section{METHODS}

This proof of concept study was performed at the Instituto Mexicano de Infertilidad in Guadalajara (Mexico), a private IVF clinic. Patients were informed about off label use of the medication. The local ethics review committee approved the study protocol and a written informed consent was obtained from all patients. Ten patients with $\mathrm{AMH}<1.0 \mathrm{nmol} / \mathrm{L}$
Recebido em 18-04-2013

Aceito em 30-07-2013
Copyright - Todos os direitos reservados a

SBRA - Sociedade Brasileira de Reprodução Assistida 
were observed over an eight months period in 2012. Patients were $22-42$ years old (average 39.3). Blood samples were drawn to determine FSH, LH, E2 and transvaginal ultrasound scans were performed on different days of the cycle. Depending on the cycle pattern of each patient, we offered individualized treatment approaches based on Natural Cycle IVF using Clomifen to control ovulation as described by Teramoto (Teramoto \& Kato, 2007), GnRH agonists to induce ovulation and either Ethinyl-Estradiol or combined oral contraceptive pills to regulate the cycle. Embryos were vitrified in day 2 stages and transferred later in artificial cycles.

\section{Study Protocol}

COCP for 10 days from day $16-26$ of cycle. Hormonal assay on day 2 of cycle: FSH, E2. If FSH >25 IU/L: Ethinyl-Estradiol $25 \mu \mathrm{g} /$ day for 7 days. If FSH $<25 \mathrm{IU} / \mathrm{L}$ : no additional treatment. Half a pill Clomifen citrate $(25 \mathrm{mg})$ from day 7 of cycle, till ovulation induction trigger. Hormonal assay on day 10 of cycle: $\mathrm{E} 2+\mathrm{LH}$. If E2 $<300 \mathrm{pg} / \mathrm{mL}$ on day 10 , E2 $+\mathrm{LH}$ was repeated every morning until E2 > $300 \mathrm{pg} / \mathrm{mL}$. Ovulation induction with $\mathrm{GnRH}$ analogues injection $0,1 \mathrm{mg}$. Egg retrieval $36 \mathrm{~h}$ after ovulation induction. Embryo vitrification 2 days after egg retrieval. Next cycle COCP for 15 days start anywhere in the cycle. GnRH analogue injection $3,75 \mathrm{mg}$ together with COCP number 10 , then 5 more days COCP. Start Estrogen valerate $4 \mathrm{mg} / \mathrm{d}$ with first day of bleeding. Vaginal ultrasound scan between day $12-14$. If endometrium $<6 \mathrm{~mm}$, Estrogen valerate $6 \mathrm{mg} / \mathrm{d}$ for 5 more days, then another vaginal ultrasound scan If endometrium $>6 \mathrm{~mm}$, start vaginal progesterone $400 \mathrm{mg} / \mathrm{d}$. Thaw and transfer 1 embryo 56 hours after initiation of vaginal progesterone.

\section{Treatment of the samples}

Sera were obtained and centrifuged for 10 minutes at $3500 \mathrm{rpm}$. Concentrations of FSH, LH and E2 were analyzed using commercially available immunoradiometric kits (Boehringer, Mannheim, Germany) and analyzed on the Elecsys immunoassay analyzer (Roche Molecular Biochemicals, Mannheim, Germany).

AMH was assayed using an ultrasensitive ELISA (AMH/ MIS Active Coated Well ELISA, DSL-10-14400, Diagnostic System Laboratories, DSL, Texas, USA). The assay range was $0.025-15 \mathrm{ng} / \mathrm{mL}$, and the functional sensitivity 0.017 $\mathrm{ng} / \mathrm{mL}$. Intra-assay and interassay coefficients of variance were $3.4 \%$ and $6.5 \%$, respectively (conversion factor to $\mathrm{pmol} / \mathrm{L}=\mathrm{ng} / \mathrm{mL} \times 7.143$ ). The manufacturer's protocol was followed in all cases.

\section{Ultrasound scans}

The ultrasound scans were performed via transvaginal probe by the same investigator.

\section{Medication}

Ethinyl-Estradiol 25 $\mu \mathrm{g}$ capsules: were prepared in a local pharmacy. GnRH analogues injections: Gonapeptyl DAILY $0,1 \mathrm{mg}$ and Gonapeptyl DEPOT 3,75mg (Ferring). Clomifen citrate pills: Omifin 50mg (Sanofi-Aventis). COCP: Yasmin (Bayer). Estradiol valerate pills: Primogyn 2mg (Bayer). Micronized Progesterone capsules: Geslutin 200mg (Asofarma).

\section{Material}

Kitazato Single Lumen needle 18G, order number 328350 , Kitazato embryo transfer catheter order number 223340. KryoTech Vitrification Kit.

\section{RESULTS}

Based on our observation, we could describe four different stages of ovarian insufficiency. We called them "compensated stage", "stage of desynchronized menstrual cycle", "stage of pathologic premature LH rise" and "stage of suppressed follicle growth." We were able to perform oocyte retrievals and embryo transfers in all 10 patients. A total of 33 natural cycles were initiated. Premature ovulation occurred in 3 cycles $(9.0 \%)$ and no retrieval was attempted. Among the attempted 30 oocyte retrievals, $21(70.0 \%)$ were successful. Out of those 21 oocytes 11 (36.6\% per retrieval) were mature and 10 (33.3\% per retrieval) were immature. ICSI resulted in 8 fertilizations ( $72.7 \%$ per mature oocyte). Out of 8 transfers, $3(37.5 \%)$ resulted in biochemical pregnancy. Two patients delivered $(25.0 \%)$, one patient had a miscarriage at 8 weeks of pregnancy.

\section{DISCUSSION}

We were able to classify four different stages of ovarian insufficiency: We called the first stage "compensated stage." In this stage the endocrine compensation mechanisms are still working and the women still have long and regular cycles of 26-33 days. Nevertheless, in some cycles the early follicular phase FSH can be elevated and the anti-mullerian Hormone (AMH) or the antral follicle count (AFC) can be low. But as long as the menstrual cycle stays long and regular, the low ovarian reserve probably does not reduce the fertility and in most of the cases there is another explanation for the infertility of the couple seeking for treatment. These patients should be treated the same way as patients with a normal ovarian reserve. The second stage of ovarian insufficiency we called "stage of desynchronized menstrual cycle." Probably due to a lower number of antral follicles in the ovaries, serum inhibin levels and other inhibiting hormones fall under a certain limit and as a consequence, basal FSH levels rise (Welt et al., 2005). Without the stabilizing effect of these hormones, the oestrogen and progesterone producing corpus luteum is not strong enough to completely block FSH production in the second half of the cycle. Due to these higher basal FSH levels in the luteal phase, follicular development starts too early, already in the middle of the luteal phase. On the onset of menses, a dominant follicle has already been recruited, which, under normal circumstances, would only happen on day six of menstrual cycle. Because of the early follicular recruitment during the luteal phase of the previous cycle, ovulation will occur earlier, and the whole cycle becomes shorter. But a shorter follicular phase may have negative effects on endometrium maturation and implantation of the embryo. Often in patients with short menstrual cycles less than 26 days, a present dominant follicle of $12-14 \mathrm{~mm}$ or more can be detected in vaginal ultrasound at the beginning of the cycle. These patients often have a normal early follicular FSH, but high early follicular estradiol levels over $60 \mathrm{pg} /$ $\mathrm{mL}$. In order to resynchronize the cycle again, we treated the patient with COCP for at least ten days during the luteal phase, for example from day 16 to day 26 of menstrual cycle. The additional effect of COCP together with the hormones produced by the corpus luteum suppresses FSH and avoids follicle formation during the luteal phase. After discontinuation of COCP, the withdrawal bleeding marks the beginning of the new, resynchronized cycle.

The third stage of ovarian insufficiency we called "stage of pathologic premature LH rise." The progression of antral follicle loss and the lack of inhibiting hormones produced by the ovaries seem to destabilize the hypothalamus - pituitary system even further in this stage. In addition to higher baseline FSH levels, the system seems to be more sensitive to oestrogen when it comes to the triggering of LH for ovulation. Under normal circumstances, the LH rise is triggered by oestrogen on the hypothalamus level, once the follicle is mature. In this stage of ovarian insufficiency, $\mathrm{LH}$ rise is triggered too early, when oestrogen levels are still low and the follicle is still small and immature. The eggs ovulated from these small and immature follicles are of a bad quality and may not lead to a pregnancy. The typical pattern in this stage are short menstrual cycles of less than 26 days, high early follicular phase FSH up to 25-30 IU/L and normal early follicular estradiol levels. Normally there is no dominant follicle at the beginning of the cycle, but nevertheless, ovulation occurs too early, due to the premature LH rise, which leads to a shorter follicular phase. The small and immature follicles seem to produce a weak corpus luteum, as we measured low progesterone during the luteal phase. The resulting luteal phase insufficiency also leads to a shorter second half of the cycle. Our way to treat the pathologic premature LH rise is to use the anti-estrogenic effect of Clomifen citrate around ovulation. As a competi- 
tive inhibitor of oestrogen receptors on the hypothalamus level, the system is tricked to "think" that the follicle is still not mature enough to trigger LH surge. Depending on the dosage, Clomifen citrate is able to delay LH surge or to block it completely (Teramoto \& Kato, 2007). In this case, it is necessary to trigger LH surge and ovulation artificially with $\mathrm{GnRH}$ analogues, once the follicle has reached maturity. It is important to understand that Clomifen citrate can be used in two different ways: either for ovarian stimulation, if given before day 6 of menstrual cycle, but in this case, it may have a negative impact on the endometrium and implantation (Wallace et al., 2011). If Clomifen citrate is given after day 6 of menstrual cycle, it has only an inhibiting effect on LH surge and ovulation, but probably no negative impact on the endometrium. In patients in the "stage of pathologic premature LH rise," the use of Clomifen citrate given around ovulation can improve follicle maturation and oocyte quality. The standard protocol in this case was $25 \mathrm{mg}$ Clomifen citrate per day from day seven of menstrual cycle including the evening of ovulation induction by $\mathrm{GnRH}$ analogues. We did not use HCG to trigger ovulation, if Clomifen citrate was used after day 6 of menstrual cycle. Clomifen citrate has a relatively long half-life and HCG somehow boosts its stimulating effect on the ovaries. If both drugs are used together, there is a higher risk for functional ovarian cysts than if $\mathrm{GnRH}$ analogues are used for triggering. Some people think that using $\mathrm{GnRH}$ analogues for triggering ovulation may cause a luteal phase defect and less good implantation, but this is only the case after ovarian stimulation with more than four mature follicles, not in a natural cycle. If the ovulation has been from a mature follicle, due to the use of Clomifen citrate, the resulting corpus luteum should be strong enough for a good luteal phase.

The fourth stage of ovarian insufficiency we called "stage of suppressed follicle growth." In this stage, either very high FSH levels of over $30 \mathrm{IU} / \mathrm{L}$, or high LH levels seem to block follicle growth, probably due to the down regulation of the respecting receptors on the follicle itself. These patients have long and irregular cycles. Most of the time the follicular growth is inhibited by the high serum FSH or LH levels, but from time to time, the ovaries seems to recover, FSH and LH levels fall to a certain limit and a follicle may grow again. Once a progesterone producing corpus luteum has developed, the ovary may enter into a dynamic phase with several ovulations during the luteal phase, as it was already described in the second stage of ovarian insufficiency. This is possible, because progesterone has a negative feedback on the hypothalamus, so FSH and $\mathrm{LH}$ are kept relatively low. But, this phenomenon of luteal phase ovulations may also lead to longer periods of amenorrhea. Every time a corpus luteum becomes weaker and is about to vanish, another one appears and progesterone production continues without withdrawal bleeding. To break the cycle of ovulations during luteal phase, we treated the patients with COCP for at least 10 days, which can be started at any time during amenorrhea. The condition is that serum progesterone levels are higher than $1.0 \mathrm{ng} /$ $\mathrm{ml}$. If no progesterone is present and $\mathrm{FSH}$ and $\mathrm{LH}$ levels are very high, there is no use of COCP treatment. In this case, a treatment with Ethinyl-Estradiol (EE) alone can be tried. In most of the cases, especially when at least one antral follicle can be seen in vaginal ultrasound, a treatment with 25 - $50 \mu \mathrm{g}$ EE over seven to ten days is sufficient to bring FSH and LH levels down to nearly physiological levels and allow the antral follicle to grow (Check et al., 1990). In order to prevent a pathologic premature LH rise, as described in the previous stage, it is useful to treat the patient additionally with $25-50$ $\mathrm{mg}$ Clomifen citrate per day, once the follicle reached $12-14 \mathrm{~mm}$ and continue this treatment till ovulation induction with $\mathrm{GnRH}$ analogues.

\section{CONCLUSION}

Our experience shows that ovulation can successfully be controlled by the use of Clomiphene citrate, as described by Teramoto (Teramoto \& Kato, 2007) and does not necessarily require $\mathrm{GnRH}$ analogues for pituitary suppression. This knowledge opens new space for development of alternative protocols respecting the patients' own physiology with no need for heavy stimulation. Patients with ovarian insufficiency may benefit from this approach, which can be offered before referring them to egg donation. In order to achieve high pregnancy rates in patients with low ovarian reserve, it is important to evaluate the patient's cycle pattern or to diagnose a possible cycle disorder. Then the treatment can be individually fixed depending on the age of the patient, her ovarian reserve and her cycle pattern. Using this empiric approach, we defined four different stages of ovarian insufficiency with the related cycle disorders. We believe that this classification may become a helpful tool for clinicians to evaluate the ovarian reserve of their patients clinically, hoping that this new approach will open a new field of investigation.

\section{Corresponding Author}

Markus Nitzschke, MD

Instituto Mexicano de Infertilidad (IMI)

Centro Medico Puerta de Hierro

Blvd. Puerta de Hierro, No. 5150

Torre C, 5th Floor, Interior 503-506

Colonia Plaza Corporativa Zapopan

Zapopan (Guadalajara), Jalisco, Mexico, C.P. 45116

Tel.: +52-322-122-2722

Fax: +52-33-3648-2550 ext.117

E-mail: markus.nitzschke@gmail.com

\section{REFERENCES}

Check JH, Nowroozi K, Chase JS, Nazari A, Shapse D, Vaze M. Ovulation induction and pregnancies in 100 consecutive women with hypergonadotropic amenorrhea. Fertil Steril. 1990;53(5):811-6.

Teramoto S, Kato O. Minimal ovarian stimulation with clomiphene citrate: a large-scale retrospective study. Reprod Biomed Online. 2007;15(2):134-48.

Wallace $\mathrm{KL}$, Johnson V, Sopelak V, Hines R. Clomiphene citrate versus letrozole: molecular analysis of the endometrium in women with polycystic ovary syndrome. Fertil Steril. 2011;96(4):1051-6.

Welt CK, Hall JE, Adams JM, Taylor AE. Relationship of estradiol and inhibin to the follicle-stimulating hormone variability in hypergonadotropic hypogonadism or premature ovarian failure. J Clin Endocrinol Metab. 2005;90(2):826-30. 